\title{
Basic Needs in Horses?-A Literature Review
}

\author{
Konstanze Krueger ${ }^{1,2}, * \mathbb{E}$, Laureen Esch ${ }^{1,3}$, Kate Farmer ${ }^{4}$ and Isabell Marr ${ }^{1,5}$ (i) \\ 1 Department of Equine Economics, Faculty of Agriculture, Economics and Management, \\ Nuertingen-Geislingen University, Neckarsteige 6-10, 72622 Nürtingen, Germany; \\ laureen.esch@gmx.de (L.E.); ISY-MARR@web.de (I.M.) \\ 2 Zoology/Evolutionary Biology, University of Regensburg, Universitätsstraße 31, 93053 Regensburg, Germany \\ 3 Department of Animal Welfare, Ethology, Animal Hygiene and Animal Husbandry, Ludwig Maximilian \\ University Munich, Veterinarian Medicine, Veterinaerstr. 13/R, 80539 Munich, Germany \\ 4 Centre for Social Learning \& Cognitive Evolution, School of Psychology, University of St Andrews, \\ St Andrews, Scotland KY16 9JPh, UK; katefarmer74@gmail.com \\ 5 Behavioural Physiology of Farm Animals, University of Hohenheim, Garbenstr. 17, \\ 70599 Hohenheim, Germany \\ * Correspondence: Konstanze.krueger@hfwu.de
}

Citation: Krueger, K.; Esch, L.; Farmer, K.; Marr, I. Basic Needs in Horses?-A Literature Review. Animals 2021, 11, 1798. https:// doi.org/10.3390/ani11061798

Academic Editors: Lisette M.C. Leliveld and Sandra Düpjan

Received: 29 April 2021

Accepted: 14 June 2021

Published: 16 June 2021

Publisher's Note: MDPI stays neutral with regard to jurisdictional claims in published maps and institutional affiliations.

\section{Copyright: (c) 2021 by the authors.} Licensee MDPI, Basel, Switzerland. This article is an open access article distributed under the terms and conditions of the Creative Commons Attribution (CC BY) license (https:// creativecommons.org/licenses/by/ $4.0 /)$.
Simple Summary: All animals have requirements that are essential for their welfare, and when these basic needs are not met, the animal suffers. In horses, it is claimed that these needs include social contact, social companionship, free movement and access to roughage in the form of grass, hay and/or straw. To validate this claim, this review examines 38 studies that reported on horses' responses when one or more of these factors are restricted. We categorised the type of responses investigated: (a) Stress (e.g., increased stress hormones), (b) Active (e.g., increased aggression), (c) Passive (e.g., depressive-like behaviour) and (d) Abnormal Behaviour (e.g., stereotypies), and analysed the frequencies with which the investigated responses were shown. Overall, the studies reported that horses did react to restrictions in the described basic needs, especially to combinations of restricted social contact, free movement and access to roughage. The observation of passive responses and the development of abnormal behaviour provided compelling evidence that horses were suffering under these restrictions, and existing abnormal behaviours indicated that they had suffered at some time in the past. We conclude that the literature supports the claim that social contact, free movement and access to roughage are basic needs in horses and need to be taken into consideration to ensure their mental and physical welfare in management and training.

Abstract: Every animal species has particular environmental requirements that are essential for its welfare, and when these so-called "basic needs" are not fulfilled, the animals suffer. The basic needs of horses have been claimed to be social contact, social companionship, free movement and access to roughage. To assess whether horses suffer when one or more of the four proposed basic needs are restricted, we examined several studies $(n=38)$ that reported behavioural and physiological reactions to these restrictions. We assigned the studies according to the four types of responses investigated: (a) Stress, (b) Active, (c) Passive, and (d) Abnormal Behaviour. Furthermore, the number of studies indicating that horses reacted to the restrictions were compared with the number of studies reporting no reaction. The limited number of studies available on single management restrictions did not allow conclusions to be drawn on the effect of each restriction separately, especially in the case of social companionship. However, when combinations of social contact, free movement and access to roughage were restricted, many of the horses had developed responses consistent with suffering. Passive Responses, indicating acute suffering, and Abnormal Behaviour, indicating suffering currently or at some time in the past, were especially clearly demonstrated. This provides further evidence of the usefulness of assessing behavioural parameters in combination with physiological measurements when evaluating horse welfare. This meta-analysis of the literature confirms that it is justified to claim that social contact, free movement and access to roughage are basic needs in horses.

Keywords: abnormal behaviour; active responses; horse; movement; passive responses; roughage; stress; social contact 


\section{Introduction}

Every animal species has particular environmental requirements that are essential for its welfare [1-3] and these are described as basic needs. As a general assumption, it has been claimed that social contact, social companionship, free movement and access to roughage are horses' basic needs [4-10]. Horses are said to need social contact because in a natural setting they live in large groups, with about $200-400$ horses comprising a herd. These herds are divided into subgroups of harems (usually composed of one to five males, several females and their offspring) and bachelor bands (composed of males of different ages) $[4,5,7,9,10]$. Furthermore, horses are assumed to need social companionship because about one third of all horses form stable social bonds with members of their subgroup. Bonded animals mutually protect each other and their offspring, as well as protecting resources such as food, water and resting places $[4,5,9]$. In addition, it has been claimed that horses need free movement because under natural conditions, they cover between 3 to $30 \mathrm{~km}$ daily [4,6-8]. Finally, horses have been said to need access to roughage as, in nature, they feed on grass for 12 to $16 \mathrm{~h}$ per day $[4,5,7,8]$.

However, it must be evaluated whether keeping horses under human management conditions that restrict their basic needs compromises welfare. Therefore, several studies have set out to assess whether horses suffer and, if so, which responses demonstrate suffering, when one or more of these needs are not fulfilled.

Many of these studies analysed whether horses reacted to such restrictions with metabolic [11-14], physiological [15-26], behavioural and/or cognitive [10,25-32] signs of stress [11-25] that would indicate reduced welfare [27]. Horses may, for example, develop gastric ulcers caused by physiological stress and when access to roughage is restricted and gastric acidosis cannot be buffered by the feed and saliva amylase secreted during feeding [27]. Stress under restricted management conditions may also affect the animals' emotional state and preferences for processing information in one or other brain hemisphere. [25,28-32]. Marr et al. [25], found that left shifts in horses' motor and sensory laterality were useful behavioural indicators of changes in information processing in particular brain hemispheres when horses experienced stress from a change from group management to individual housing with initial training. Pioneering comparisons between hemispheric electroencephalogram (EEG) wave patterns in horses and those typical of emotional arousal in humans provide further evidence that horses respond negatively to restrictions in movement and social contact [26]. Furthermore, Löckener et al. [10] found that horses develop positive expectations towards their environment, i.e., a positive cognitive bias, when moved to group housing after experiencing management restriction.

Increased displays of certain behaviours in response to restrictions in basic needs have also been evaluated. Horses may show increased aggressive behaviour towards each other $[18,22,33,34]$ and/or towards people [26,35], especially when their social relationships are disrupted [11,18,36-38]. Horses may show more interest in novel objects [38-40] and increased cooperativeness during training (i.e., trainability) when they are stabled in social groups rather than individually $[13,21]$. They may seek close proximity to their conspecifics more frequently when their social companionship is disrupted, as has been evaluated by applying nearest neighbour analysis [36]. Furthermore, horses may become more active when free movement, either individually or in social groups, is restricted $[14,15,19,21,24,38,39]$, or when foals are weaned and separated from dams and social companions [40]. They may also show more hurried eating behaviour when roughage is limited [14]. On the other hand, horses may respond to restrictions in basic needs by reducing certain behaviour displays [11,15,18-22,24,26,35,38-43] and may even show depressive-type symptoms [22].

Behavioural disorders have also been assessed in horses [17,44], including stereotypies $[8,26,45-48]$ and self-harming (i.e., redirected behaviour [8]). It has been debated whether abnormal behaviour is actually harmful or is rather a behavioural adaptation to a poor environment [47]. In this respect, most authors agree that stereotypies can be considered maladaptive behaviour indicating that horses are suffering under their housing or training conditions, and they give various reasons for this conclusion. Firstly, horses 
develop stereotypies in housing in which movement is reduced and there is little social contact. Secondly, free ranging, feral horses have never, to date, been observed showing stereotypic behaviour [7]. Thirdly, animals that displayed redirected behaviour and stereotypies also showed clinical signs of reduced welfare. These included: (a) self-inflicted skin lesions as a result of redirected behaviours, (b) gut damage, including lesions, ulceration and damaged mucosal tissue as a consequence of a wind-sucking and crib-biting [27,49] and (c) increased frequency of laminitis as a result of stereotypic movements such as weaving and box walking [27].

This literature review aims to provide an overview of studies that have evaluated the effects of changes in factors relating to horses' environmental requirements (specifically social contact, social companionship, free movement, and access to roughage) on behavioural or physiological parameters (Table 1). We asked (a) whether a meta-analysis of the relevant literature supports the claim that social contact, social companionship, free movement and unlimited access to roughage are basic needs in horses, and (b) whether certain measurements can be considered reliable indicators for the analysis of animal welfare and basic need restrictions.

Table 1. Literature on restrictions in "proposed basic needs" in horses. Background colours indicate studies on the restricted basic needs: grey $=$ no restriction, light orange $=$ social contact, light blue = social companionship, light yellow $=$ movement, dark grey $=$ feed, orange $=$ social contact and feed, green $=$ movement and social contact, yellow = movement, social contact and feed. The columns list the test condition or changes in test conditions, the response measurements and the horses' responses that were observed. For detailed information on the horses and their management conditions please see Supplementary Materials (Table S1).

\begin{tabular}{|c|c|c|c|c|}
\hline Reference & Restriction & $\begin{array}{l}\text { Condition(s) } \\
\text { Observed }\end{array}$ & Measurements & Response(s) \\
\hline $\begin{array}{l}\text { Hoffmann } \\
\text { et al. } 2012 \text { [11] }\end{array}$ & $\begin{array}{l}\text { No } \\
\text { restriction }\end{array}$ & $\begin{array}{l}\text { Group housing-no other } \\
\text { conditions }\end{array}$ & $\begin{array}{l}\text { Body condition score } \\
\text { Behaviour: aggression } \\
\text { Behaviour: social hierarchy } \\
\text { Behaviour: synchronisation }\end{array}$ & $\begin{array}{l}\text { Good } \\
\text { Low } \\
\text { Stable } \\
\text { Good }\end{array}$ \\
\hline \multirow{4}{*}{$\begin{array}{l}\text { Christensen } \\
\text { et al. } 2002 \text { [36] }\end{array}$} & \multirow{4}{*}{$\begin{array}{l}\text { Social } \\
\text { contact }\end{array}$} & \multirow{4}{*}{$\begin{array}{l}\text { Behaviour of young horses } \\
\text { that had either been raised in } \\
\text { group housing or single } \\
\text { boxes were compared with } \\
\text { behaviour when the horses } \\
\text { were put out to pasture with } \\
\text { other horses of the same age }\end{array}$} & Nearest neighbour & $\begin{array}{l}\text { Horses from group housing } \\
\text { sought proximity to former } \\
\text { stable mates } \\
\text { Higher in previously singly } \\
\text { housed horses }\end{array}$ \\
\hline & & & $\begin{array}{l}\text { Behaviour: agonistic } \\
\text { encounters (action and } \\
\text { retreat response) }\end{array}$ & $\begin{array}{l}\text { More subtle encounters in } \\
\text { previously group housed horses }\end{array}$ \\
\hline & & & Behaviour: social grooming & $\begin{array}{l}\text { More frequent in previously } \\
\text { singly housed horses }\end{array}$ \\
\hline & & & Behaviour: play & $\begin{array}{l}\text { More frequent in previously } \\
\text { singly housed horses }\end{array}$ \\
\hline $\begin{array}{l}\text { Cooper et al. } \\
2000[33]\end{array}$ & $\begin{array}{l}\text { Social } \\
\text { contact }\end{array}$ & $\begin{array}{l}\text { Comparison between } \\
\text { different types of single box } \\
\text { housing: } \\
\text { F: front top-half of the door } \\
\text { open with a view of the } \\
\text { stable courtyard FB: front } \\
\text { half-door open and a similar } \\
\text { half-door open at the back of } \\
\text { the stable with a view to the } \\
\text { surrounding fields } \\
\text { B: back open only } \\
\text { FS: front and one-side panel } \\
\text { open with a view into the } \\
\text { adjacent stable ALL } 4 \text { : front, } \\
\text { back and both sides open }\end{array}$ & $\begin{array}{l}\text { Stereotypies: weaving } \\
\text { Stereotypies: repetitive } \\
\text { nodding }\end{array}$ & $\begin{array}{l}\text { Most common prior to feeding in } \\
\text { the morning and prior to putting } \\
\text { out to pasture in the afternoon. } \\
\text { Less weaving in the FS and All4 } \\
\text { designs than the F design } \\
\text { FB, B, FS and All4: less nodding } \\
\text { than in the F treatment }\end{array}$ \\
\hline
\end{tabular}


Table 1. Cont

\begin{tabular}{|c|c|c|c|c|}
\hline Reference & Restriction & $\begin{array}{l}\text { Condition(s) } \\
\text { Observed }\end{array}$ & Measurements & Response(s) \\
\hline $\begin{array}{l}\text { Hartmann } \\
2010[18]\end{array}$ & $\begin{array}{l}\text { Social } \\
\text { contact }\end{array}$ & $\begin{array}{l}5 \text { min social isolation from } \\
\text { group housing (individually } \\
\text { or in pairs) }\end{array}$ & $\begin{array}{l}\text { Heart rate } \\
\text { Behaviour: towards humans }\end{array}$ & $\begin{array}{l}\text { No change } \\
\text { No change }\end{array}$ \\
\hline $\begin{array}{l}\text { Nicol et al. } \\
2005 \text { [40] }\end{array}$ & $\begin{array}{l}\text { Social } \\
\text { contact }\end{array}$ & $\begin{array}{l}\text { Comparison between barn } \\
\text { and paddock weaned foals }\end{array}$ & Stress & $\begin{array}{l}\text { Higher levels of stress in barn } \\
\text { weaned foals }\end{array}$ \\
\hline $\begin{array}{l}\text { Christensen } \\
\text { et al. } 2011 \text { [50] }\end{array}$ & $\begin{array}{l}\text { Social com- } \\
\text { panionship }\end{array}$ & $\begin{array}{l}\text { Comparison between } \\
\text { housing in unstable } \\
\text { (changing) groups and stable } \\
\text { (constant) groups }\end{array}$ & $\begin{array}{l}\text { Behaviour: agonistic } \\
\text { Behaviour: agonistic with } \\
\text { contact } \\
\text { Behaviour: greeting } \\
\text { Behaviour: play } \\
\text { Behaviour: agonistic (further } \\
\text { behaviours) } \\
\text { Behaviour: affiliative (further } \\
\text { behaviours) }\end{array}$ & $\begin{array}{l}\text { More in unstable groups } \\
\text { More in unstable groups } \\
\text { More in unstable groups } \\
\text { More variable in unstable groups } \\
\text { No difference between housing } \\
\text { groups } \\
\text { No difference between housing } \\
\text { groups }\end{array}$ \\
\hline $\begin{array}{l}\text { Chaplin and } \\
\text { Gretgrix } 2010 \\
\text { [39] }\end{array}$ & Movement & $\begin{array}{l}\text { Same horses compared } \\
\text { under Fully stabled (FS), } \\
\text { Partially stabled (PS), Yard } \\
(\mathrm{Y}) \text {, and Paddock (P) }\end{array}$ & $\begin{array}{l}\text { Activity: time spent active } \\
\text { Activity: time spent lying } \\
\text { down }\end{array}$ & $\begin{array}{l}\text { More active on release from FS } \\
\text { and PS housing } \\
\text { No change }\end{array}$ \\
\hline $\begin{array}{l}\text { Flauger and } \\
\text { Krueger } 2013 \\
{[34]}\end{array}$ & Movement & $\begin{array}{l}\text { Different sizes of group } \\
\text { paddock }\end{array}$ & $\begin{array}{l}\text { Behaviour: aggressive } \\
\text { Behaviour: submissive }\end{array}$ & $\begin{array}{l}\text { Decreased with increasing size } \\
\text { of group paddock } \\
\text { Decreased with increasing size } \\
\text { of group paddock }\end{array}$ \\
\hline $\begin{array}{l}\text { Hoffmann } \\
\text { et al. } 2009 \text { [20] }\end{array}$ & Movement & $\begin{array}{l}\text { Provision of additional } \\
\text { movement on pasture or in } \\
\text { horse walker }\end{array}$ & $\begin{array}{l}\text { Stress: faecal glucocorticoids } \\
\text { Stress: heart rate variability } \\
\text { Activity: movement }\end{array}$ & $\begin{array}{l}\text { Decreased after movement } \\
\text { Decrease of sympathetic activity } \\
\text { after movement } \\
\text { Increased }\end{array}$ \\
\hline $\begin{array}{l}\text { McGreevy } \\
\text { et al. 1995a } \\
\text { [45] }\end{array}$ & Movement & $\begin{array}{l}\text { Length of time spent in } \\
\text { single box housing }\end{array}$ & Stereotypic behaviour & $\begin{array}{l}\text { Increased with time spent in } \\
\text { individual boxes }\end{array}$ \\
\hline $\begin{array}{l}\text { Brinkmann } \\
\text { et al. } 2013 \text { [51] }\end{array}$ & Feed & Feed restriction & $\begin{array}{l}\text { Body condition score } \\
\text { Total bilirubin } \\
\text { NEFA } \\
\text { Total bilirubin and } \\
\text { beta-hydroxyburyrat } \\
\text { Thyroxine concentrations }\end{array}$ & $\begin{array}{l}\text { Decreased } \\
\text { Increased } \\
\text { Increased } \\
\text { Higher in males than in females } \\
\text { No change }\end{array}$ \\
\hline $\begin{array}{l}\text { Brinkmann } \\
\text { et al. } 2014 \text { [52] }\end{array}$ & Feed & $\begin{array}{l}\text { Metabolic adaptation to } \\
\text { environmental conditions, } \\
\text { comparing different } \\
\text { quantities of feed and } \\
\text { summer and winter } \\
\text { temperatures }\end{array}$ & $\begin{array}{l}\text { Body condition score } \\
\text { Body mass } \\
\text { Resting heart rate } \\
\text { Metabolic rate } \\
\text { Nocturnal hypothermia }\end{array}$ & $\begin{array}{l}\text { Reduced in winter } \\
\text { Reduced in winter } \\
\text { Reduced in winter } \\
\text { Reduced in winter } \\
\text { Increased in winter }\end{array}$ \\
\hline $\begin{array}{l}\text { McGreevy } \\
\text { et al. } 1995 \mathrm{~b} \\
{[46]}\end{array}$ & Feed & $\begin{array}{l}\text { Stabled without straw } \\
\text { bedding and with less feed } \\
\text { than } 6.8 \mathrm{~kg} \text { forage/day }\end{array}$ & Abnormal behaviour & Increased \\
\hline $\begin{array}{l}\text { Jørgensen } \\
\text { et al. } 2011 \text { [42] }\end{array}$ & $\begin{array}{l}\text { Social } \\
\text { contact \& } \\
\text { feed }\end{array}$ & $\begin{array}{l}\text { Single turnout on paddocks } \\
\text { versus group turnout. } \\
\text { Feeding grass and roughage }\end{array}$ & $\begin{array}{l}\text { Behaviour: passive } \\
\text { Behaviour: passive } \\
\text { Behaviour: item exploration }\end{array}$ & $\begin{array}{l}\text { Increased when turned out } \\
\text { singly } \\
\text { Reduced when fed with } \\
\text { roughage or grass } \\
\text { No difference }\end{array}$ \\
\hline $\begin{array}{l}\text { Aurich et al. } \\
2015[23]\end{array}$ & $\begin{array}{l}\text { Movement } \\
\& \text { social } \\
\text { contact }\end{array}$ & $\begin{array}{l}\text { Group versus individual } \\
\text { housing }\end{array}$ & Stress: salivary cortisol & No significant difference \\
\hline
\end{tabular}


Table 1. Cont

\begin{tabular}{|c|c|c|c|c|}
\hline Reference & Restriction & $\begin{array}{l}\text { Condition(s) } \\
\text { Observed }\end{array}$ & Measurements & Response(s) \\
\hline $\begin{array}{l}\text { Erber et al. } \\
2013 \text { [19] }\end{array}$ & $\begin{array}{l}\text { Movement } \\
\& \text { social } \\
\text { contact }\end{array}$ & $\begin{array}{l}\text { Transfer from group housing } \\
\text { to individual housing with } \\
\text { initial riding }\end{array}$ & $\begin{array}{l}\text { Stress: salivary cortisol } \\
\text { Stress: heart rate } \\
\text { Stress: heat rate variability } \\
\text { Activity: locomotion } \\
\text { Stress: salivary cortisol } \\
\text { Stress: heart rate }\end{array}$ & $\begin{array}{l}\text { Increase after transfer } \\
\text { Increase during transfer } \\
\text { Decrease after transfer and after } \\
\text { riding (= increase of sympathetic } \\
\text { activity) } \\
\text { Decrease after transfer } \\
\text { No change between housing } \\
\text { conditions } \\
\text { No change between housing } \\
\text { conditions }\end{array}$ \\
\hline $\begin{array}{l}\text { Fureix et al. } \\
2012 \text { [22] }\end{array}$ & $\begin{array}{l}\text { Movement } \\
\& \text { social } \\
\text { contact }\end{array}$ & $\begin{array}{l}\text { Horses showing normal and } \\
\text { horses showing withdrawn } \\
\text { posture under conditions of } \\
\text { no free movement and no } \\
\text { free social contact }\end{array}$ & $\begin{array}{l}\text { Stress: plasma cortisol } \\
\text { Activity: body posture } \\
\text { Activity: head, ear, eye } \\
\text { movement } \\
\text { Activity: response to tactile } \\
\text { stimuli } \\
\text { Activity: response to sudden } \\
\text { approaching person } \\
\text { Activity: response to novel } \\
\text { objects }\end{array}$ & $\begin{array}{l}\text { Low after work-further decrease } \\
\text { with increased withdrawn } \\
\text { posture } \\
\text { Withdrawn posture 1-4 times } \\
\text { every } 30 \text { min } \\
\text { Reduced in withdrawn posture } \\
\text { Reduced in withdrawn posture } \\
\text { Reduced in withdrawn posture } \\
\text { Reduced in withdrawn posture }\end{array}$ \\
\hline $\begin{array}{l}\text { Harewood } \\
\text { and } \\
\text { McGowan } \\
2005 \text { [16] }\end{array}$ & $\begin{array}{l}\text { Movement } \\
\& \text { social } \\
\text { contact }\end{array}$ & $\begin{array}{l}\text { Group versus individual } \\
\text { housing }\end{array}$ & $\begin{array}{l}\text { Behavioural scores } \\
\text { Stress: heart rate } \\
\text { Stress: salivary cortisol } \\
\text { Diurnal rhythm heart rate } \\
\text { and salivary cortisol }\end{array}$ & $\begin{array}{l}\text { Higher in individual than in } \\
\text { group housing } \\
\text { No difference } \\
\text { No difference } \\
\text { No diurnal rhythm under either } \\
\text { condition }\end{array}$ \\
\hline $\begin{array}{l}\text { Heleski et al. } \\
2002 \text { [12] }\end{array}$ & $\begin{array}{l}\text { Movement } \\
\text { \& social } \\
\text { contact }\end{array}$ & $\begin{array}{l}\text { Paddock-kept weanlings } \\
\text { versus stable housed } \\
\text { weanlings }\end{array}$ & $\begin{array}{l}\text { Nearest neighbour } \\
\text { Activity: grazing } \\
\text { Behaviours } \\
\text { Abnormal behaviour } \\
\text { Stress: faecal glucocorticoid } \\
\text { metabolites }\end{array}$ & $\begin{array}{l}\text { Paddock weaned foals stayed } \\
\text { near conspecifics for longer } \\
\text { Higher in paddock weaned foals } \\
\text { Greater variety in paddock } \\
\text { weaned foals } \\
\text { Greater in stable weaned foals } \\
\text { No difference }\end{array}$ \\
\hline $\begin{array}{l}\text { Löckener et al. } \\
2016 \text { [10] }\end{array}$ & $\begin{array}{l}\text { Movement } \\
\& \text { social } \\
\text { contact }\end{array}$ & $\begin{array}{l}\text { Living at pasture with social } \\
\text { contact following single box } \\
\text { housing }\end{array}$ & $\begin{array}{l}\text { Behaviour: positive cognitive } \\
\text { bias }\end{array}$ & $\begin{array}{l}\text { Enhanced in horses on pasture } \\
\text { with social contact }\end{array}$ \\
\hline $\begin{array}{l}\text { Niederhöfer } \\
2009 \text { [17] }\end{array}$ & $\begin{array}{l}\text { Movement } \\
\text { \& social } \\
\text { contact }\end{array}$ & $\begin{array}{l}\text { Comparison between group } \\
\text { housing, single box without } \\
\text { paddock, and single box } \\
\text { with paddock }\end{array}$ & $\begin{array}{l}\text { Stress: faecal glucocorticoid } \\
\text { metabolites } \\
\text { Stress: heart rate variability } \\
\text { Abnormal behaviour }\end{array}$ & $\begin{array}{l}\text { Lower in group housing } \\
\text { Lower in group housing } \\
\text { Circling in horses in single boxes } \\
\text { without paddock }\end{array}$ \\
\hline $\begin{array}{l}\text { Pell and } \\
\text { McGreevy } \\
1999[44]\end{array}$ & $\begin{array}{l}\text { Movement } \\
\& \text { social } \\
\text { contact }\end{array}$ & $\begin{array}{l}\text { Stable housing compared to } \\
\text { keeping at pasture }\end{array}$ & Abnormal behaviour & More frequent in stabled horses \\
\hline
\end{tabular}


Table 1. Cont

\begin{tabular}{|c|c|c|c|c|}
\hline Reference & Restriction & $\begin{array}{c}\text { Condition(s) } \\
\text { Observed }\end{array}$ & Measurements & Response(s) \\
\hline $\begin{array}{l}\text { Rivera et al. } \\
2002 \text { [15] }\end{array}$ & $\begin{array}{l}\text { Movement } \\
\text { \& social } \\
\text { contact }\end{array}$ & $\begin{array}{l}\text { Stable housing versus } \\
\text { keeping at pasture }\end{array}$ & $\begin{array}{l}\text { Stress: heart rate } \\
\text { Illness: gastric acidosis } \\
\text { Trainability: duration } \\
\text { training procedure } \\
\text { Trainability: duration } \\
\text { habituation groundwork } \\
\text { Trainability: head neck } \\
\text { extension during training } \\
\text { Behaviour: bucking and } \\
\text { jumping } \\
\text { Stress: plasma cortisol } \\
\text { Trainability: between mount } \\
\text { and dismount }\end{array}$ & $\begin{array}{l}\text { Lower in stabled horses } \\
\text { More frequent in stabled horses } \\
\text { Longer in stabled horses } \\
\text { Longer in stabled horses } \\
\text { Greater in stabled horses } \\
\text { More frequent in stabled horses } \\
\text { No difference } \\
\text { No effect of housing conditions }\end{array}$ \\
\hline $\begin{array}{l}\text { Ruet et al. } \\
2019 \text { [43] }\end{array}$ & $\begin{array}{l}\text { Movement } \\
\text { \& social } \\
\text { contact }\end{array}$ & $\begin{array}{l}\text { Housing with window } \\
\text { opening towards the external } \\
\text { environment and straw } \\
\text { bedding compared with } \\
\text { housing with no window } \\
\text { and non-straw bedding, } \\
\text { different forage: grain }\end{array}$ & $\begin{array}{l}\text { Behaviour: aggression } \\
\text { Stereotypies: oral } \\
\text { Stereotypies: oral } \\
\text { Trainability: equitation and } \\
\text { training }\end{array}$ & $\begin{array}{l}\text { Lower in housing with window } \\
\text { and straw bedding } \\
\text { Higher with grain feeding } \\
\text { Number of meals per day had no } \\
\text { effect } \\
\text { No difference }\end{array}$ \\
\hline $\begin{array}{l}\text { Sondergaard } \\
\text { and Ladewig } \\
2004 \text { [35] }\end{array}$ & $\begin{array}{l}\text { Movement } \\
\text { \& social } \\
\text { contact }\end{array}$ & $\begin{array}{l}\text { Effect of single versus group } \\
\text { housing on training }\end{array}$ & $\begin{array}{l}\text { Activity: restlessness before } \\
\text { training } \\
\text { Behaviour: biting, kicking } \\
\text { during training } \\
\text { Behaviour: defecation during } \\
\text { training } \\
\text { Trainability }\end{array}$ & $\begin{array}{l}\text { Greater in single housed horses } \\
\text { More frequent in horses in single } \\
\text { housing } \\
\text { More frequent in horses in single } \\
\text { housing } \\
\text { Horses in group housing passed } \\
\text { more training stages }\end{array}$ \\
\hline $\begin{array}{l}\text { Vitale et al. } \\
2013[53]\end{array}$ & $\begin{array}{l}\text { Movement } \\
\text { \& social } \\
\text { contact }\end{array}$ & $\begin{array}{l}\text { paddock turnout versus } \\
\text { individual box housing } \\
\text { versus fixed in a stock }\end{array}$ & Stress: heart rate variability & $\begin{array}{l}\text { Decreased with reduced } \\
\text { locomotion (= increased } \\
\text { sympathetic activity) }\end{array}$ \\
\hline $\begin{array}{l}\text { Werhahn et al. } \\
2011[38]\end{array}$ & $\begin{array}{l}\text { Movement } \\
\text { \& social } \\
\text { contact }\end{array}$ & $\begin{array}{l}\text { No turnout compared to } \\
\text { turnout }\end{array}$ & $\begin{array}{l}\text { Behaviours: standing alert, } \\
\text { aggression, occupation with } \\
\text { equipment, occupation with } \\
\text { bedding, dozing, sternal } \\
\text { recumbency and lateral } \\
\text { recumbency } \\
\text { Activity: walking, } \\
\text { standing/watching } \\
\text { Trainability: willingness to } \\
\text { perform } \\
\text { Trainability: duration of } \\
\text { training } \\
\text { Locomotion }\end{array}$ & $\begin{array}{l}\text { More frequent in the horses with } \\
\text { no turnout } \\
\text { More frequent in the horses with } \\
\text { no turnout } \\
\text { Enhanced in horses with turnout } \\
\text { Shorter in horses with turnout } \\
\text { No difference }\end{array}$ \\
\hline $\begin{array}{l}\text { Werhahn et al. } \\
2012 \text { [21] }\end{array}$ & $\begin{array}{l}\text { Movement } \\
\text { \& social } \\
\text { contact }\end{array}$ & $\begin{array}{l}\text { Single box housing, } \\
\text { individual turnout, group } \\
\text { turnout }\end{array}$ & $\begin{array}{l}\text { Stress: heart rate variability } \\
\text { measures SDNN, RMSSD } \\
\text { and LF/HF } \\
\text { Behaviour: lying down } \\
\text { Trainability: willingness to } \\
\text { perform } \\
\text { Behaviour: standing alert, } \\
\text { dozing, eating, occupation } \\
\text { Locomotion }\end{array}$ & $\begin{array}{l}\text { Higher sympathetic activity } \\
\text { when horses were stabled in } \\
\text { single boxes } \\
\text { Longer when horses had group } \\
\text { turnout } \\
\text { Slightly better when the horses } \\
\text { had turnout } \\
\text { No change } \\
\text { No change }\end{array}$ \\
\hline
\end{tabular}


Table 1. Cont.

\begin{tabular}{|c|c|c|c|c|}
\hline Reference & Restriction & $\begin{array}{l}\text { Condition(s) } \\
\text { Observed }\end{array}$ & Measurements & Response(s) \\
\hline Wille 2010 [24] & $\begin{array}{l}\text { Movement } \\
\text { \& social } \\
\text { contact }\end{array}$ & $\begin{array}{l}\text { Open barn housing, } \\
\text { individual box housing, tied } \\
\text { up in stalls }\end{array}$ & $\begin{array}{l}\text { Stress: faecal glucocorticoid } \\
\text { metabolites } \\
\text { Behaviour: standing } \\
\text { Behaviour: lying on the chest } \\
\text { Behaviour: lying on the side } \\
\text { Locomotion } \\
\text { Behaviour: food } \\
\text { consumption }\end{array}$ & $\begin{array}{l}\text { Lower in open barn system } \\
\text { Longer when tied in stalls } \\
\text { Longer in open barn system or } \\
\text { individual boxes } \\
\text { Longer in open barn system } \\
\text { More in open barn system } \\
\text { No difference }\end{array}$ \\
\hline $\begin{array}{l}\text { Yarnell et al. } \\
2015 \text { [13] }\end{array}$ & $\begin{array}{l}\text { Movement } \\
\& \text { social } \\
\text { contact }\end{array}$ & $\begin{array}{l}\text { Single housing with no } \\
\text { contact (SHNC), group } \\
\text { housing with full contact } \\
\text { (GHFC), paired housing with } \\
\text { full contact (PHFC) }\end{array}$ & $\begin{array}{l}\text { Stress: faecal glucocorticoid } \\
\text { metabolites } \\
\text { Stress: eye temperature } \\
\text { Behaviour: standing } \\
\text { Behaviour: active and social } \\
\text { negative behaviours } \\
\text { Trainability: handling }\end{array}$ & $\begin{array}{l}\text { Higher in SHNC } \\
\text { Lower in GHFC } \\
\text { Lower in GHFC } \\
\text { Higher in GHFC and PHFC } \\
\text { More difficult with SHNC horses }\end{array}$ \\
\hline $\begin{array}{l}\text { Lesimple et al. } \\
2020[54]\end{array}$ & $\begin{array}{l}\text { Movement } \\
\& \text { feed }\end{array}$ & $\begin{array}{l}\text { Change from single box with } \\
\text { no paddock to housing with } \\
\text { turnout and ad lib hay }\end{array}$ & $\begin{array}{l}\text { Behaviour: vigilance, } \\
\text { excitement and locomotion } \\
\text { Behaviour: feeding with ears } \\
\text { laid back } \\
\text { Stereotypies } \\
\text { Blood: oxytocin } \\
\text { Blood: cell counts, serotonin }\end{array}$ & $\begin{array}{l}\text { Decreased with turnout and ad } \\
\text { lib hay } \\
\text { Decreased with turnout and ad } \\
\text { lib hay } \\
\text { Decreased with turnout and ad } \\
\text { lib hay } \\
\text { Increased with turnout and ad } \\
\text { lib hay } \\
\text { No change }\end{array}$ \\
\hline $\begin{array}{l}\text { Bachmann } \\
\text { et al. } 2003 \text { [48] }\end{array}$ & $\begin{array}{l}\text { Movement, } \\
\text { social contact } \\
\text { \& feed }\end{array}$ & $\begin{array}{l}\text { Restricting feed and daily } \\
\text { pasture }\end{array}$ & $\begin{array}{l}\text { Stereotypies: crib-biting, } \\
\text { weaving and box-walking }\end{array}$ & Increased \\
\hline & & & $\begin{array}{l}\text { Behaviour: time eating grain, } \\
\text { grain-eating bouts }\end{array}$ & $\begin{array}{l}\text { More in horses of medium and } \\
\text { highly reactive temperaments in } \\
\text { isolation (ISS) }\end{array}$ \\
\hline $\begin{array}{l}\text { Mal et al. } 1991 \\
{[14]}\end{array}$ & $\begin{array}{l}\text { Movement, } \\
\text { social } \\
\text { contact \& } \\
\text { feed }\end{array}$ & $\begin{array}{l}\text { Horses of different } \\
\text { temperaments; housing in } \\
\text { isolation (ISS), at pasture (P), } \\
\text { in individual boxes with } \\
\text { social contact (C) }\end{array}$ & $\begin{array}{l}\text { Behaviour: forage-eating } \\
\text { bouts } \\
\text { Activity: distance travelled, } \\
\text { time spent trotting, number } \\
\text { of trotting bouts, number of } \\
\text { standing bouts, number of } \\
\text { total activity bouts } \\
\text { Activity: duration standing } \\
\text { Triiodothyronine }\end{array}$ & $\begin{array}{l}\text { More in isolation horses } \\
\text { Less in isolation horses } \\
\text { Highest in isolation horses }\end{array}$ \\
\hline $\begin{array}{l}\text { Marr et al. } \\
2020[25]\end{array}$ & $\begin{array}{l}\text { Movement, } \\
\text { social } \\
\text { contact \& } \\
\text { feed }\end{array}$ & $\begin{array}{l}\text { Change from group housing } \\
\text { to individual housing, and } \\
\text { initial training }\end{array}$ & $\begin{array}{l}\text { Behaviour: motor laterality } \\
\text { Behaviour: sensory laterality }\end{array}$ & $\begin{array}{l}\text { Increased after change from } \\
\text { group to individual housing } \\
\text { after } 24 \mathrm{~h}, 48 \mathrm{~h} \text {, and } 1 \text { week. } \\
\text { Increased after } 24 \mathrm{~h}, 48 \mathrm{~h} \text {, and } 2 \\
\text { months of initial training } \\
\text { Left shift for } 1 \text { week after change } \\
\text { from group to individual } \\
\text { housing, and after } 2 \text { months of } \\
\text { initial training } \\
\text { Left shift } 24 \mathrm{~h} \text { after change from } \\
\text { group to individual housing, } \\
\text { and (not significantly) } 24 \mathrm{~h} \text { after } \\
\text { initial training } \\
\text { Decreased (not significantly) } \\
\text { after change from group to } \\
\text { individual housing, and } 24 \mathrm{~h} \\
\text { after initial training }\end{array}$ \\
\hline
\end{tabular}


Table 1. Cont.

\begin{tabular}{|c|c|c|c|c|}
\hline Reference & Restriction & $\begin{array}{l}\text { Condition(s) } \\
\text { Observed }\end{array}$ & Measurements & Response(s) \\
\hline \multirow{2}{*}{$\begin{array}{l}\text { Redbo et al. } \\
1998 \text { [55] }\end{array}$} & \multirow{2}{*}{$\begin{array}{l}\text { Movement, } \\
\text { social contact } \\
\text { \& Feed }\end{array}$} & \multirow{2}{*}{$\begin{array}{l}\text { Thoroughbreds compared } \\
\text { with trotters }\end{array}$} & Behaviour: wood-chewing & No difference \\
\hline & & & Stereotypies & More in thoroughbreds \\
\hline \multirow{9}{*}{$\begin{array}{l}\text { Stomp et al. } \\
2021[26]\end{array}$} & \multirow{9}{*}{$\begin{array}{l}\text { Movement, } \\
\text { social } \\
\text { contact \& } \\
\text { feed }\end{array}$} & \multirow{9}{*}{$\begin{array}{l}\text { Hemispheric activity in } \\
\text { horses in individual housing } \\
\text { compared with that in horses } \\
\text { kept at pasture }\end{array}$} & $\begin{array}{l}\text { Electroencephalogram (EEG): } \\
\text { bilateral predominance of } \\
\text { theta waves }\end{array}$ & Increased in pasture kept horses. \\
\hline & & & & $\begin{array}{l}\text { Increased in horses in individual } \\
\text { housing }\end{array}$ \\
\hline & & & EEG: hemispheric laterality: & \\
\hline & & & $\begin{array}{l}\text { bilateral and } \\
\text { Left-Hemispheric theta }\end{array}$ & Increased in pasture kept horses \\
\hline & & & activity & \\
\hline & & & $\begin{array}{l}\text { EEG: hemispheric laterality: } \\
\text { bilateral or } \\
\text { Right-Hemispheric high } \\
\text { production of gamma waves }\end{array}$ & $\begin{array}{l}\text { Increased in horses in individual } \\
\text { housing }\end{array}$ \\
\hline & & & Stereotypies & $\begin{array}{l}\text { More common in horses in } \\
\text { individual housing }\end{array}$ \\
\hline & & & $\begin{array}{l}\text { Behaviour: ear position } \\
\text { while feeding }\end{array}$ & $\begin{array}{l}\text { More common in horses in } \\
\text { individual housing }\end{array}$ \\
\hline & & & $\begin{array}{l}\text { Behaviour: human } \\
\text { approach-tests }\end{array}$ & $\begin{array}{l}\text { Pasture kept horses more } \\
\text { positive }\end{array}$ \\
\hline \multirow{4}{*}{$\begin{array}{l}\text { Visser et al. } \\
2008[41]\end{array}$} & \multirow{4}{*}{$\begin{array}{l}\text { Movement, } \\
\text { social } \\
\text { contact \& } \\
\text { feed }\end{array}$} & \multirow{4}{*}{$\begin{array}{l}\text { Housing in individual boxes } \\
\text { versus housing in pairs }\end{array}$} & $\begin{array}{l}\text { Stress: CRF challenge } \\
\text { test-cortisol response and } \\
\text { ACTH response }\end{array}$ & $\begin{array}{l}\text { Lower in individually housed } \\
\text { horses }\end{array}$ \\
\hline & & & $\begin{array}{l}\text { Behaviour: neighing, } \\
\text { pawing, nibbling, snorting }\end{array}$ & $\begin{array}{l}\text { More frequent in individually } \\
\text { housed horses }\end{array}$ \\
\hline & & & Stereotypies & $\begin{array}{l}\text { More frequent in individually } \\
\text { housed horses }\end{array}$ \\
\hline & & & Activity: novel object test & No difference \\
\hline \multirow{2}{*}{$\begin{array}{l}\text { Waters et al. } \\
2002[8]\end{array}$} & \multirow{2}{*}{$\begin{array}{l}\text { Movement, } \\
\text { social contact } \\
\& \text { feed }\end{array}$} & \multirow{2}{*}{$\begin{array}{l}\text { Weaning in a stable, a barn, } \\
\text { on a paddock, and at grass }\end{array}$} & Abnormal behaviour & $\begin{array}{l}\text { More frequent after weaning in } \\
\text { barns or stables }\end{array}$ \\
\hline & & & $\begin{array}{l}\text { Stereotypic behaviour: wood } \\
\text { chewing }\end{array}$ & $\begin{array}{l}\text { More frequent after weaning in } \\
\text { barns or stables }\end{array}$ \\
\hline
\end{tabular}

\section{Materials and Methods}

From August 2020 to February 2021, we searched the research platforms Research Gate, PubMed, Web of Science, Science Direct and Google Scholar for studies on social contact, social companionship, free movement and unlimited access to roughage in horses. We identified 38 studies (Table 1 and Table S1) on behavioural and physiological responses to management conditions in which one or more of the four proposed basic needs were restricted, and this will be the basis of this literature review. Information on the horses observed in the studies and the management conditions they lived under are given in the Supplementary Materials (Table S1). Of the studies we identified, 17 evaluated horses' responses to the given management situation, and 21 studied horses' responses to changes in management conditions. One study is cited, but not included in the analysis, as it describes the horses' responses to particular management conditions but does not analyse responses to management restrictions.

As horse housing is a complex setting, isolating only one of the aspects is very difficult. Therefore, only a few studies have analysed changes in only one of the proposed basic needs, and most consider two or more needs simultaneously (see Table 1). These generally compared two or more horse groups under housing conditions that differed in one or more needs (see Table 1), but a few examined changes in housing conditions of one group. 


\subsection{Data Processing}

Four steps were applied in the evaluation of the literature (Table 1 and Figure 1). Firstly, the literature was assigned to the proposed basic needs that were restricted. Secondly, the studies were categorized according to the type of response shown by the horse, and thirdly, we assessed whether changes in the behavioural and physiological reactions indicated that the restrictions were compromising the horses' welfare. Finally, the frequencies of studies reporting responses were compared with the frequency of those reporting no responses to the restrictions.

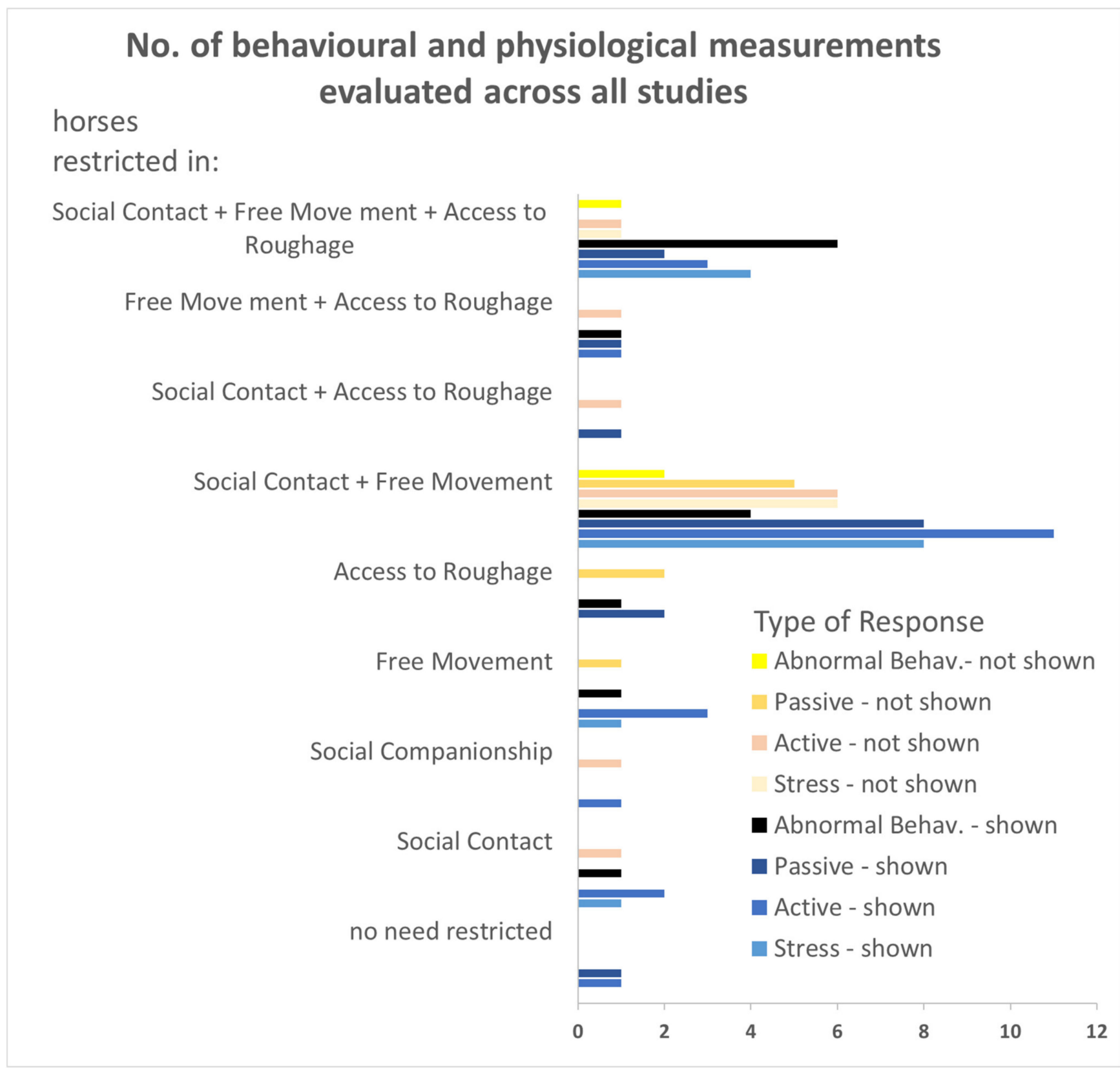

Figure 1. Number of reports of responses (dark colours, i.e., black and blue) versus number of reports of no response (light colours: skin and yellow) to restrictions in proposed "basic needs" described for response types across all studies. Abnormal Behaviour responses were significantly shown across all studies (Binomial Test: $p=0.02$ ). Responses were also shown when social contact, free movement and access to roughage were mutually restricted (Binomial Test: $p<0.001$ ). More detailed information on the responses, the test conditions and the horses included in the studies are given in Table 1 and in the Supplementary Materials (Table S1). 
The studies were grouped according to the proposed basic needs that were restricted (Table 1):

1. No basic need restricted, $n=1$

2. Social Contact restricted, $n=4$

3. Social Companionship restricted, $n=1$

4. Free Movement restricted, $n=4$

5. Access to Roughage restricted, $n=3$

6. Social Contact and Access to Roughage restricted, $n=1$

7. Free Movement and Social Contact restricted, $n=16$

8. Free Movement and Access to Roughage restricted, $n=1$

9. Free Movement, Social Contact and Access to Roughage restricted, $n=7$

The studies were grouped according to evaluated behavioural and physiological measurements. The terms "shown" and "not shown" indicates whether the horses displayed any of the following types of responses to management conditions or not (Table 1, Figure 1):

a. Manuscripts evaluating 'Stress Responses', $n=16$, methods of measuring 'Stress Responses', $n=21$

i. shown, $n=14$

ii. not shown, $n=7$

Stress responses include increased metabolic rates and reduced body condition score [11], reduced growth rate [12], reduced eye temperature [13] and triiodothyronine and thyroxine excretion [14]. Physiological responses include changes in cardiovascular functions (heart rate [15-19], heart rate variability [19-21]), excretion of catecholamines [14], changes in stress hormone levels (blood cortisol [14,15,22], salivary cortisol [16,19,23], faecal glucocorticoid metabolites [12,13,17,20,24,25]), faecal immunoglobulin A [25], increased gastric acidosis [27] and changes in EEG wave patterns [26]. Behavioural parameters comprise changes in horses' motor and sensory laterality [25,28-32] and changes in their positive or negative expectations towards their environment, i.e., in their cognitive bias [10].

b. Manuscripts evaluating 'Active Responses', $n=24$, methods of measuring 'Active Responses', $n=33$

i. shown, $n=22$

ii. not shown, $n=11$

Horses may show increased aggressive behaviour towards each other and/or towards people [11,18,22,26,33-38]. They may show more interest in novel objects [38-40] and increased cooperativeness during training (i.e., trainability) $[13,21]$. They may seek close proximity to their conspecifics more frequently, as observed by applying nearest neighbour analysis [36]. Furthermore, horses may become more active [14,15,19,21,24,38-40] and may also show more hurried eating behaviour [14].

c. Manuscripts evaluating 'Passive Responses', $n=17$, methods of measuring 'Passive Responses', $n=23$

i. shown, $n=15$

ii. not shown, $n=8$

Passive responses include reduced reactivity towards stimuli and human presence [15, $22,26,40,41]$, reduced close contact with conspecifics [35] and reduced trainability [15,35,43]. Reduced activity [42] was evaluated by measuring the time spent lying down [11,21,39], moving [11,19], and the distance moved [20]. Some horses may show depressive-like behaviours [22].

d. Manuscripts evaluating 'Abnormal Behaviour', $n=14$, methods of measuring 'Abnormal Behaviour', $n=17$

i. shown, $n=14$

ii. not shown, $n=3$ 
Abnormal Behaviours include self-harming (i.e., redirected behaviour such as selfbiting [8]), stereotypies such as crib-biting, wind-sucking, weaving and box-walking [14,41,48], as well as other behaviours such as, wood-chewing, bed-eating, manure-eating, rugchewing or tearing, stable kicking, aggression towards humans and masturbation $[8,44,55]$.

\subsection{Data Analysis}

The R-Project statistical software (R Development Core Team 2021, https: / /www.rproject.org /) was used for the statistical analysis and Excel for creating the figures when analysing the frequencies of studies on basic need restrictions. Some of the data were not normally distributed (Shapiro-Wilk Test). Therefore, Generalized Linear Models (GLMs) for multivariate testing with fixed factors were applied. For a general approach, we analysed the frequency of studies reporting reactions or no reaction under the particular restrictions of basic needs and the different responses shown. The GLM (formula = number of manuscripts evaluating response response versus no response + response type, family = poisson (identity), data = Dataset) was used. We continued by applying a nested Generalized Linear Model (GLM) to analyse whether manuscripts reported differences for showing or not showing responses nested within the different types of response. Therefore, the GLM (formula = number of manuscripts. evaluating response $\sim$ response versus no response $\%$ in $\%$ response type, family = poisson (identity), data = Dataset) was applied. The full statistical data are given in the Supplementary Materials (File S1). Binomial Tests were applied to compare the frequencies of showing or not showing certain types of responses under certain management restrictions. All tests were two-tailed, and the significance level was set at 0.05 .

\section{Results}

Overall, a significant number of studies reported that horses did show responses to restrictions in the proposed basic needs (responses shown: $n=90$, not shown: $n=29$; GLM: $n=37, \mathrm{z}=4.08, p<0.001$; Table 1, Figure 1, File S1). However, reports on whether horses showed responses differed between the particular response types (GLM: $n=37, \mathrm{z}=-2.57$, $p=0.01$; Figure 1, Table 1, File S1).

Of the studies on changes in behavioural and physiological measurements of 'Stress Responses', $n=13$ studies reported that horses showed responses and $n=7$ did not (Table 1 , Figure 1). The studies did not clearly indicate stress responses for single restrictions, as when studies found several stress measurements changed, i.e., indicating stress, the same study or other studies also found other stress measurements remained unchanged, i.e., indicating no stress. When the reactions to all the different restrictions were compared, the number of reports showing stress responses correlated with the number of reports showing no response (GLM: $n=37, \mathrm{z}=2.49, p=0.01$; Figure 1, Table 1, File S1).

The same was true for the 'Active Responses', with $n=14$ studies reporting that active responses were shown and $n=8$ reporting they were not (Figure 1 , Table 1). Again, while many parameters changed almost as many did not change when analysing a particular restriction and there was a general correlation between active responses and no active response over all the basic need restrictions (GLM: $n=37, \mathrm{z}=2.63$; $p=0.008$, Figure 1 , Table 1, File S1).

'Passive Responses' were reported in $n=14$ studies and no 'Passive Responses' for $n=8$ studies (Figure 1, Table 1). 'Passive Responses' to particular restrictions indicated more clearly than 'Stress Responses' and 'Active Responses' that horses responded with changes in behaviour to certain restrictions. The literature revealed only a trend in correlation between the horses showing passive responses to those showing no passive response when comparing all the different restrictions in the basic needs (GLM: $n=37, \mathrm{z}=1.85, p=$ 0.06; Figure 1, Table 1, File S1).

Finally, a significant number of horses demonstrated the response 'Abnormal Behaviour' in response to any restriction in basic needs ('Abnormal Behaviour': $n=12$, no 'Abnormal Behaviour': $n=3$, Binomial test: $p=0.02$, Figure 1). Horses reacted more clearly 
by either showing abnormal behaviour or not showing abnormal behaviour to particular restrictions, as there was again only a trend for a correlation between reports of abnormal behaviour and reports of no abnormal behaviour across the studies on all the different restrictions examined (GLM: $n=37, \mathrm{z}=1.74, p=0.08$; Figure 1, Table 1, File S1).

The literature provides no clear conclusion as to whether horses generally show responses to restrictions in just one of the parameters of social contact, social companionship, free movement and access to roughage in isolation (Figure 1). For most of the comparisons the sample sizes were too small for applying statistical tests, the few that allowed statistical testing revealed no difference between measurements that show and those that do not show a response to the restrictions (Binomial tests: all $p>0.05$; Figure 1). When social contact, free movement and access to roughage were mutually restricted, horses showed behavioural and/or physiological responses (Binomial test: $p<0.001$; Figure 1).

\section{Discussion}

The aim of this study was to evaluate whether certain measurements can be considered reliable indicators for the analysis of animal welfare under basic need restrictions by analysing the results published in the literature. The interpretation of the studies calls for caution as positive reporting biases may result in more studies that prove a certain measurement to be effective than those reporting no effect [56]. However, the literature also contains several studies that actually report missing responses of horses to the measurements under observation. We therefore found the results of the meta analysis of these studies worth discussing.

Especially the development of abnormal behaviour and stress responses under the long-term stress of compromised environmental conditions has been considered a maladaptive strategy which does not provide the animals with options to deal with such conditions $[8,57,58]$. When animals develop these responses, they are said to be clearly suffering $[58,59]$. The present literature review provides evidence that this claim is justified in horses, especially in relation to abnormal behaviour. Horses develop significant levels of abnormal behaviour when social contact, social companionship, free movement and access to roughage are compromised. Therefore, it appears to be justified to use the display of abnormal behaviour as a behavioural animal welfare indicator when analysing the quality of horse housing and training, as has been established for animal welfare protocols [60].

However, it remains debatable whether a long-term display of abnormal behaviour provides strong evidence of a horse suffering under its present management conditions. Stereotypic behaviour may have developed under previous conditions and persist, even when management and training return to favourable conditions [45,46,57]. A recent development in EEG wave pattern analysis [26] offers promising new insights into this debate. Horses that had consistently displayed stereotypic behaviour for at least one year and lived under restricted management conditions showed EEG wave patterns comparable to those indicating negative emotional states in humans. However, caution should be exercised when evaluating welfare in horses displaying stereotypic behaviour as horses secrete dopamine when engaging in such behaviour and this elicits a positive emotional state in the animal (see for review: [61]).

Similarly, the measurement of behavioural and physiological stress parameters did not necessarily indicate that all horses experienced stress in compromised management conditions (Figure 1). Some studies found that horses did respond with behavioural or physiological stress parameters, and others did not (Table 1). This discrepancy may be due to difficulties in assessing long-term stress. When animals suffer stress for longer periods, such as when their environmental requirements are restricted for a long time, several physiological parameters, such as stress hormones and cardiovascular functions may return to base levels or below $[13,25,62,63]$.

There are promising stress parameters that may allow long-term stress to be evaluated, such as immune cell suppression, changes in motor laterality [25,64], increased hemispheric laterality and EEG wave patterns analogous to those measured in humans with negative 
emotional arousal [26]. However, there is not yet sufficient evidence for these to be included in the present literature survey because they have only been studied in a few pioneering papers $[25,26,64,65]$.

There may also be individual differences between horses in their stress resistance and the importance an individual attaches to any particular stressor [63]. In addition, previous experience with the restrictions in basic needs may be a factor. For example, horses that were born and raised in a stabled environment may be less stressed by restricted movement than horses raised at grass and then moved to a stabled environment [25]. Moreover, horses that had previously experienced individual housing did not display any significant differences in physiological stress responses between individual housing with semi-contact to conspecifics and group housing [13,24], whereas horses that were naïve to individual housing showed significant physiological stress responses when moved from group to individual housing $[17,19,25]$. A combination of several physiological and behavioural stress parameters may provide the strongest evidence for stress in horses as some studies found conflicting results when comparing a limited spectrum of physiological and behavioural data [63].

However, the proportion of horses showing passive responses supports the claim that many horses suffer long term stress under the investigated management restrictions [22, 52,66]. Passive responses, such as reductions in activity, feeding, behaviour displays, contact to persons or other conspecifics and reactions to the environment indicate that horses withdraw from external stimuli and may show a depressive-like state [22,66]. These responses are maladaptive for animals such as horses, which are both flight animals that rely on fast responses to acute challenges and social animals that rely on fast responses to social challenges [9].

Interestingly, the analysis of 'Active Responses' did not provide a clear conclusion. Some studies reported that horses showed active responses when the animals were faced with restricted basic needs and others did not (Table 1 and Table S1). It may be difficult to clearly distinguish the level of activity that constitutes a stress response, as elevated aggression and movement may counteract mild stress [34,37].

As only very few studies succeeded in isolating the effects of restrictions in just one of the four proposed basic needs of social contact, social companionship, free movement and access to roughage, a clear statement on whether animals can generally cope with the particular restrictions remains elusive. However, there were sufficient studies on combined restrictions in social contact, free movement and access to roughage, and when the horses' environments were restricted in these three conditions, they appeared to suffer, as 'Abnormal Behaviour' and 'Passive Response' had clearly developed. This is consistent with the finding that abnormal behaviour display may be caused by a variety factors (e.g., genetics, rearing conditions, housing and feeding [48]).

\section{Conclusions}

We conclude that under combined restrictions of social contact, social companionship, free movement and access to roughage horses display signs of suffering. The literature available on individual so called "basic needs", does not allow us to isolate the effect of each of them, especially in the case of social companionship. However, the development of abnormal behaviour and passive coping strategies, can be considered signs of suffering, and these were displayed under separate restrictions in social contact, free movement and free access to roughage, as well as under combined restrictions of two or more of the proposed basic needs.

Supplementary Materials: The following are available online at https:/ /www.mdpi.com/article/10 .3390/ani11061798/s1, File S1: Statistical Data. Complete Generalized Linear Models (GLMs), Table S1: Summary table of literature content. More detailed information on the restrictions, the horses, their management and their responses. 
Author Contributions: Each author made substantial contributions to the conception or design of the work. Individual contributions were done for Conceptualization, K.K., L.E. and I.M.; Methodology, K.K., L.E. and I.M.; Software, K.K.; Validation, K.K., L.E. and I.M.; Formal Analysis, K.K.; Resources, K.K., L.E. and I.M.; Data Curation, K.K., L.E. and I.M.; Writing-Original Draft Prep-aration, K.K., L.E., K.F. and I.M.; Writing-Review \& Editing, K.K., L.E., K.F. and I.M.; Visualization, K.K., L.E. and I.M.; Funding Acquisition, K.K. Each author has approved the submitted version (and versions substantially edited by journal staff that involves the author's contribution to the study); and agrees to be personally accountable for the author's own contributions and for ensuring that questions related to the accuracy or integrity of any part of the work, even ones in which the author was not personally involved, are appropriately investigated, resolved, and documented in the literature. All authors have read and agreed to the published version of the manuscript.

Funding: The article processing charge was funded by the Baden-Württemberg Ministry of Science, Research and Culture and the Nuertingen-Geislingen University in the funding programme Open Access Publishing.

Institutional Review Board Statement: Ethical review and approval were waived for this study because it did not involve humans or animals.

Informed Consent Statement: No humans or animals were involved in the study.

Data Availability Statement: All data are provided in the manuscript and the Supplementary Material.

Conflicts of Interest: The authors have declared that no competing interests exist.

\section{References}

1. Noë, R.; Hammerstein, P. Biological markets. Trends Ecol. Evol. 1995, 10, 336-339. [CrossRef]

2. Pusey, A.E.; Packer, C. The Ecology of relationships. In Behavioural Ecology; Krebs, J.R., Davis, N.B., Eds.; Blackwell Scientific Publication Oxford: Hoboken, NJ, USA, 2003; pp. 254-283.

3. Korb, J.; Heinze, J. Ecology of Social Evolution; Springer: Berlin/Heidelberg, Germany, 2008; ISBN 9783540759560.

4. Berger, J. Wild Horses of the Great Basin; University of Chicago Press: Chicago, IL, USA, 1986.

5. Rubenstein, D.I. Ecology and sociality in horses and zebras. In Ecological Aspects of Social Evolution; Rubenstein, D.I., Wrangham, R.W., Eds.; Princeton University Press: Princeton, NJ, USA, 1986; pp. 282-302.

6. Mills, D.; Clarke, A. Housing, Management and Welfare. In The Welfare of Horses; Waran, N., Ed.; Springer Publishing: New York, NY, USA, 2002; pp. 77-97.

7. Goodwin, D. Horse behaviour: Evolution, domestication and feralisation. In The Welfare of Horses; Waran, N., Ed.; Springer Publishing: New York, NY, USA, 2002; pp. 1-18.

8. Waters, A.J.; Nicol, C.; French, N.P. Factors influencing the development of stereotypic and redirected behaviours in young horses: Findings of a four year prospective epidemiological study. Equine Vet. J. 2010, 34, 572-579. [CrossRef] [PubMed]

9. Krueger, K. Social Ecology of Horses. In Ecology of Social Evolution; Korb, J., Heinze, J., Eds.; Springer: Berlin/Heidelberg, Germany, 2008; pp. 195-206.

10. Löckener, S.; Reese, S.; Erhard, M.H.; Wöhr, A.-C. Pasturing in herds after housing in horseboxes induces a positive cognitive bias in horses. J. Vet. Behav. 2016, 11, 50-55. [CrossRef]

11. Hoffmann, G.; Bentke, A.; Rose-Meierhöfer, S.; Berg, W.; Mazetti, P.; Hardarson, G.H. Influence of an active stable system on the behavior and body condition of Icelandic horses. Animal 2012, 6, 1684-1693. [CrossRef] [PubMed]

12. Heleski, C.; Shelle, A.; Nielsen, B.; Zanella, A. Influence of housing on weanling horse behavior and subsequent welfare. Appl. Anim. Behav. Sci. 2002, 78, 291-302. [CrossRef]

13. Yarnell, K.; Hall, C.; Royle, C.; Walker, S.L. Domesticated horses differ in their behavioural and physiological responses to isolated and group housing. Physiol. Behav. 2015, 143, 51-57. [CrossRef] [PubMed]

14. Mal, M.E.; Friend, T.H.; Lay, D.C.; Vogelsang, S.G.; Jenkins, O.C. Behavioural responses of mares to short-term confinement and social isolation. Appl. Anim. Behav. Sci. 1991, 31, 13-24. [CrossRef]

15. Rivera, E.; Benjamin, S.; Nielsen, B.; Shelle, J.; Zanella, A. Behavioral and physiological responses of horses to initial training: The comparison between pastured versus stalled horses. Appl. Anim. Behav. Sci. 2002, 78, 235-252. [CrossRef]

16. Harewood, E.; McGowan, C. Behavioral and physiological responses to stabling in naive horses. J. Equine Vet. Sci. 2005, 25, 164-170. [CrossRef]

17. Niederhöfer, S. Stressbelastung bei Pferden in Abhängigkeit des Haltungssystems. Ph.D. Thesis, Tierärztliche Hochschule Hannover, Hannover, Germany, 2009.

18. Hartmann, E. Managing Horses in Groups to Improve Horse Welfare and Human Safety. Ph.D. Thesis, Swedish University of Agricultural Sciences, Uppsala, Sweden, 2010. 
19. Erber, R.; Wulf, M.; Aurich, J.; Rose-Meierhöfer, S.; Hoffmann, G.; Von Lewinski, M.; Möstl, E.; Aurich, C. Stress Response of Three-year-old Horse Mares to Changes in Husbandry System During Initial Equestrian Training. J. Equine Vet. Sci. 2013, 33, 1088-1094. [CrossRef]

20. Hoffmann, G.; Bockisch, F.-J.; Kreimeier, P. Einfluss des Haltungssystems auf die Bewegungsaktivität und Stressbelastung bei Pferden in Auslaufhaltungssystemen [Influence of the husbandry system on the movement activity and stress exposure of horses in discharge husbandry systems]. Agric. For. Res. 2009, 2, 105-112.

21. Werhahn, H.; Hessel, E.F.; Van den Weghe, H.F. Competition Horses Housed in Single Stalls (II): Effects of Free Exercise on the Behavior in the Stable, the Behavior during Training, and the Degree of Stress. J. Equine Vet. Sci. 2012, 32, 22-31. [CrossRef]

22. Fureix, C.; Bourjade, M.; Henry, S.; Sankey, C.; Hausberger, M. Exploring aggression regulation in managed groups of horses Equus caballus. Appl. Anim. Behav. Sci. 2012, 138, 216-228. [CrossRef]

23. Aurich, J.; Wulf, M.; Ille, N.; Erber, R.; Von Lewinski, M.; Palme, R.; Aurich, C. Effects of season, age, sex, and housing on salivary cortisol concentrations in horses. Domest. Anim. Endocrinol. 2015, 52, 11-16. [CrossRef]

24. Wille, E. Vergleichende Untersuchungen von Pferden in Zeitweiliger Anbindehaltung, Einzelboxenhaltung und Gruppenhal-tung unter Besonderer Berücksichtigung des Liegeverhaltens; Tierärztliche Hochschule: Hannover, Germany, 2010.

25. Marr, I.; Preisler, V.; Farmer, K.; Stefanski, V.; Krueger, K. Non-invasive stress evaluation in domestic horses (Equus caballus): Impact of housing conditions on sensory laterality and immunoglobulin A. R. Soc. Open Sci. 2020, 7, 191994. [CrossRef]

26. Stomp, M.; D'Ingeo, S.; Henry, S.; Cousillas, H.; Hausberger, M. Brain activity reflects (chronic) welfare state: Evidence from individual electroencephalography profiles in an animal model. Appl. Anim. Behav. Sci. 2021, 236, 105271. [CrossRef]

27. Rowe, J.B.; Lees, M.J.; Pethick, D.W. Prevention of Acidosis and Laminitis Associated with Grain Feeding in Horses. J. Nutr. 1994, 124, 2742S-2744S. [CrossRef]

28. LaRose, C.; Richard-Yris, M.-A.; Hausberger, M.; Rogers, L.J. Laterality of horses associated with emotionality in novel situations. Laterality 2006, 11, 355-367. [CrossRef]

29. De Boyer Des Roches, A.; Richard-Yris, M.-A.; Henry, S.; Ezzaouïa, M.; Hausberger, M. Laterality and emotions: Visual laterality in the domestic horse (Equus caballus) differs with objects' emotional value. Physiol. Behav. 2008, 94, 487-490. [CrossRef]

30. Rogers, L.J. Relevance of brain and behavioural lateralization to animal welfare. Appl. Anim. Behav. Sci. 2010, 127, 1-11. [CrossRef]

31. Marr, I.; Farmer, K.; Krüger, K. Evidence for Right-Sided Horses Being More Optimistic than Left-Sided Horses. Animals 2018, 8 , 219. [CrossRef] [PubMed]

32. Giljov, A.; Karenina, K. Differential roles of the right and left brain hemispheres in the social interactions of a free-ranging ungulate. Behav. Process. 2019, 168, 103959. [CrossRef] [PubMed]

33. Cooper, J.; McDonald, L.; Mills, D.S. The effect of increasing visual horizons on stereotypic weaving: Implications for the social housing of stabled horses. Appl. Anim. Behav. Sci. 2000, 69, 67-83. [CrossRef]

34. Flauger, B.; Krueger, K. Aggression level and enclosure size in horses (Equus caballus). Pferdeheilkunde 2013, 29, 495-504. [CrossRef]

35. Søndergaard, E.; Ladewig, J. Group housing exerts appositive effect on the behaviour of young horses during training. Appl. Anim. Behav. Sci. 2004, 87, 105-118. [CrossRef]

36. Christensen, J.W.; Ladewig, J.; Søndergaard, E.; Malmkvist, J. Effects of individual versus group stabling on social behaviour in domestic stallions. Appl. Anim. Behav. Sci. 2002, 75, 233-248. [CrossRef]

37. Knubben, J.M.; Fürst, A.; Gygax, L.; Stauffacher, M. Bite and kick injuries in horses: Prevalence, risk factors and prevention. Equine Vet. J. 2008, 40, 219-223. [CrossRef]

38. Werhahn, H.; Hessel, E.F.; Schulze, H.; Weghe, H.F.V.D. Temporary Turnout for Free Exercise in Groups: Effects on the Behavior of Competition Horses Housed in Single Stalls. J. Equine Vet. Sci. 2011, 31, 417-425. [CrossRef]

39. Chaplin, S.; Gretgrix, L. Effect of housing conditions on activity and lying behaviour of horses. Animal 2010, 4, 792-795. [CrossRef]

40. Nicol, C.; Badnell-Waters, A.; Bice, R.; Kelland, A.; Wilson, A.; Harris, P. The effects of diet and weaning method on the behaviour of young horses. Appl. Anim. Behav. Sci. 2005, 95, 205-221. [CrossRef]

41. Visser, E.K.; Ellis, A.D.; Van Reenen, C.G. The effect of two different housing conditions on the welfare of young horses stabled for the first time. Appl. Anim. Behav. Sci. 2008, 114, 521-533. [CrossRef]

42. Jørgensen, G.H.M.; Liestøl, S.H.-O.; Bøe, K.E. Effects of enrichment items on activity and social interactions in domestic horses (Equus caballus). Appl. Anim. Behav. Sci. 2011, 129, 100-110. [CrossRef]

43. Ruet, A.; LeMarchand, J.; Parias, C.; Mach, N.; Moisan, M.-P.; Foury, A.; Briant, C.; Lansade, L. Housing Horses in Individual Boxes Is a Challenge with Regard to Welfare. Animals 2019, 9, 621. [CrossRef]

44. Pell, S.M.; McGreevy, P. Prevalence of stereotypic and other problem behaviours in thoroughbred horses. Aust. Vet. J. 1999, 77, 678-679. [CrossRef]

45. McGreevy, P.; French, N.P.; Nicol, C.J. The prevalence of abnormal behaviours in dressage, eventing and endurance horses in relation to stabling. Vet. Rec. 1995, 137, 36-37. [CrossRef]

46. McGreevy, P.D.; Cripps, P.J.; French, N.P.; Green, L.E.; Nicol, C.J. Management factors associated with stereotypic and redi-rected behaviour in the thoroughbred horse. Equine Vet. J. 1995, 27, 86-91. [CrossRef]

47. Cooper, J.J.; Mason, G.J. The identification of abnormal behaviour and behavioural problems in stabled horses and their rela-tionship to horse welfare: A comparative review. Equine Vet. J. 1998, 27, 5-9.

48. Bachmann, I.; Audigé, L.; Stauffacher, M. Risk factors associated with behavioural disorders of crib-biting, weaving and box-walking in Swiss horses. Equine Vet. J. 2010, 35, 158-163. [CrossRef] 
49. Nicol, C. Equine learning: Progress and suggestions for future research. Appl. Anim. Behav. Sci. 2002, 78, 193-208. [CrossRef]

50. Christensen, J.W.; Søndergaard, E.; Thodberg, K.; Halekoh, U. Effects of repeated regrouping on horse behaviour and injuries. Appl. Anim. Behav. Sci. 2011, 133, 199-206. [CrossRef]

51. Brinkmann, L.; Gerken, M.; Riek, A. Effect of long-term feed restriction on the health status and welfare of a robust horse breed, the Shetland pony (Equus ferus caballus). Res. Vet. Sci. 2013, 94, 826-831. [CrossRef] [PubMed]

52. Brinkmann, L.; Gerken, M.; Hambly, C.; Speakman, J.R.; Riek, A. Saving energy during hard times: Energetic adaptations of Shetland pony mares. J. Exp. Biol. 2014, 217, 4320-4327. [CrossRef] [PubMed]

53. Vitale, V.; Balocchi, R.; Varanini, M.; Sgorbini, M.; Macerata, A.; Sighieri, C.; Baragli, P. The effects of restriction of movement on the reliability of heart rate variability measurements in the horse (Equus caballus). J. Vet. Behav. 2013, 8, 400-403. [CrossRef]

54. Lesimple, C.; Reverchon-Billot, L.; Galloux, P.; Stomp, M.; Boichot, L.; Coste, C.; Henry, S.; Hausberger, M. Free movement: A key for welfare improvement in sport horses? Appl. Anim. Behav. Sci. 2020, 225, 104972. [CrossRef]

55. Redbo, I.; Redbo-Torstensson, P.; Ödberg, F.O.; Hedendahl, A.; Holm, J. Factors affecting behavioural disturbances in race-horses. Anim. Sci. 1998, 66, 475-481. [CrossRef]

56. Dwan, K.; Altman, D.G.; Arnaiz, J.A.; Bloom, J.; Chan, A.-W.; Cronin, E.; Decullier, E.; Easterbrook, P.J.; von Elm, E.; Gamble, C.; et al. Systematic Review of the Empirical Evidence of Study Publication Bias and Outcome Reporting Bias. PLoS ONE 2008, 3, e3081. [CrossRef]

57. Nicol, C. Understanding equine stereotypies. Equine Vet. J. 2010, 31, 20-25. [CrossRef]

58. Kupriyanov, R.; Zhdanov, R. The eustress concept: Problems and outlooks. World J. Med. Sci. 2014, 11, $179-185$.

59. Cooper, J.; Albentosa, M.J. Behavioural adaptation in the domestic horse: Potential role of apparently abnormal responses including stereotypic behaviour. Livest. Prod. Sci. 2005, 92, 177-182. [CrossRef]

60. Costa, E.D.; Dai, F.; Lebelt, D.; Scholz, P.; Barbieri, S.; Canali, E.; Minero, M. Welfare assessment of horses: The AWIN approach. Anim. Welf. 2016, 25, 481-488. [CrossRef]

61. McBride, S.D.; Parker, M.; Roberts, K.; Hemmings, A. Applied neurophysiology of the horse; implications for training, husbandry and welfare. Appl. Anim. Behav. Sci. 2017, 190, 90-101. [CrossRef]

62. Pawluski, J.; Jego, P.; Henry, S.; Bruchet, A.; Palme, R.; Coste, C.; Hausberger, M. Low plasma cortisol and faecal cortisol metabolite measures as indicators of compromised welfare in domestic horses (Equus caballus). PLoS ONE 2017, 12, e0182257. [CrossRef]

63. Von Borstel, U.K.; Visser, E.; Hall, C. Indicators of stress in equitation. Appl. Anim. Behav. Sci. 2017, 190, 43-56. [CrossRef]

64. May, A. Evaluierung von Stressparametern beim Pferd im Zusammenhang mit dem Klinikaufenthalt [Evaluation of Stress Parameters in the Hospitalized Horse]. Ph.D. Thesis, Ludwig Maximilians University (LMU), München, Germany, 2007.

65. Rogers, L.J. A Matter of Degree: Strength of Brain Asymmetry and Behaviour. Symmetry 2017, 9, 57. [CrossRef]

66. Fureix, C.; Jego, P.; Henry, S.; Lansade, L.; Hausberger, M. Towards an Ethological Animal Model of Depression? A Study on Horses. PLoS ONE 2012, 7, e39280. [CrossRef] 